\title{
Psg18 Is Specifically Expressed in Follicle-associated Epithelium
}

\author{
Kazuya Kawano ${ }^{1, \dagger}$, Masashi Ebisawa ${ }^{1,3, \dagger}$, Koji Hase $^{1}$, Shinji Fukuda $^{1}$, Atsushi Hijikata ${ }^{2}$, \\ Sayaka Kawano", Yasuhiro Date ${ }^{1,5}$, Satoshi Tsuneda ${ }^{5}$, Kikuji Itoh ${ }^{4}$, and Hiroshi Ohno ${ }^{1,3^{*}}$ \\ ${ }^{1}$ Laboratory for Epithelial Immunobiology, Research Center for Allergy and Immunology, RIKEN, \\ Kanagawa, Japan, ${ }^{2}$ Laboratory for immunogenomics, Research Center for Allergy and Immunology, RIKEN, \\ Kanagawa, Japan, ${ }^{3}$ International Graduate School of Arts and Sciences, Yokohama City University, \\ Kanagawa, Japan, ${ }^{4}$ Graduate School of Agricultural and Life Sciences, The University of Tokyo, Bunkyo-ku, \\ Tokyo, Japan, ${ }^{5}$ Graduate School of Advanced Science and Engineering, Waseda University, Shinjuku-ku, \\ Tokyo, Japan
}

\begin{abstract}
Pregnancy-specific glycoproteins (Psgs) secreted by the placenta regulate the immune system to ensure the survival of the fetal allograft by inducing IL-10, an anti-inflammatory cytokine. However, it is unknown whether Psgs are involved in more general aspects of immune response other than maternal immunity. Here, we report that Psg18 is highly expressed in the follicle-associated epithelium (FAE) overlaying Peyer's patches (PPs). Bioinformatics analysis with Reference Database for Immune Cells (RefDIC) as well as RT-PCR data demonstrated that Psg18 is exclusively expressed in FAE in adult mice, in contrast to other Psg family members that are either not expressed or only slightly expressed in FAE. Psg18 expression was observed in FAE of germ-free-conditioned mice, and was slightly upregulated after bacterial inoculation. In situ hybridization analysis revealed that Psg18 is widely expressed throughout FAE. Furthermore, Psg18 protein is deposited on the extracellular matrix in the subepithelial dome beneath FAE, where antigen-presenting cells accumulate. These results suggest that Psg18 is an FAE-specific marker protein that could promote interplay between FAE and immune cells in mucosa-associated lymphoid tissues.
\end{abstract}

Key words: Pregnancy-specific glycoprotein 18/FAE/Peyer's patch/mucosal immunity/microarray

\section{Introduction}

Epithelial cells lining the mucosal surface are an important mechanical barrier that separates the host's internal milieu from the external environment. In addition to their function as a physical barrier, epithelial cells, sensing microorganism invasion and inflammatory signals on the mucosa, rapidly release an array of chemokines toward the immune cells of the lamina propria to initiate an immune response (Sansonetti, 2004). Recent studies have demonstrated that homeostasis of the mucosal immune system is regulated by the interaction between epithelial cells and immunocytes. For instance, treatment of dendritic cells (DCs) with the culture supernatant of intestinal epithelial cells resulted in the induction of anti-inflammatory cytokines, interleukin (IL)-10 and IL-6,

\footnotetext{
*To whom correspondence should be addressed: Hiroshi Ohno, Laboratory of Epithelial Immunobiology, Research Center for Allergy and Immunology, RIKEN, 1-7-22 Suehirocho, Tsurumi-ku, Yokohama, Kanagawa 230-0045, Japan.

Tel: +81-45-503-7032, Fax: +81-45-503-7030

E-mail: ohno@rcai.riken.jp

$\dagger$ Kazuya Kawano, Masashi Ebisawa: These two authors contributed equally to this work.
}

but not IL-12, in response to Salmonella infection (Rimoldi et al., 2005). This effect is mediated by thymic stromal lymphopoietin (TSLP) and other factors released by the intestinal epithelium.

Lymph-epithelial interaction seems to be particularly important for FAE. Previous studies on the genetic profiling of FAE have demonstrated that a number of host-defense molecules, including antimicrobial peptides and subsets of chemokines, are upregulated in FAE compared to normal villous epithelium (Hase et al., 2005). This suggests that FAE may be immunologically activated by signals from immune cells present in SED and lymphoid follicles underneath, and in turn, chemokines secreted by FAE are critical for the recruitment and retention of lymphocytes and DCs in SED, and should be responsible for immunocyte compartmentalization in PPs (Hase et al., 2006; Zhao et al., 2003). This positive feedback loop underscores the importance of crosstalk between epithelium and immune cells for the maintenance of the microarchitecture in gut-associated lymphoid tissues (GALT).

$\mathrm{CD}^{+} \mathrm{T}$ cells localized in PPs are characterized by their capacity to induce Th2 (IL-4 and IL-10) and Th3 (trans- 
forming growth factor; TGF- $\beta$ ) cytokines (Chen et al., 1995b). These Th2/Th3 responses are essential for the production of IgA and the induction of oral tolerance. Multiple studies have proposed that the microenvironment in PPs, including cytokine balance and properties of resident antigen-presenting cells, favors the development of Th2/Th3 cells. This concept is supported by the fact that antigen presentation by freshly isolated PP DCs, but not splenic DCs, promotes the secretion of Th2 cytokines by naïve $\mathrm{T}$ cells (Iwasaki and Kelsall, 1999). However, it is largely unknown what factors confer such a functional phenotype on PP DCs. We hypothesized that certain immunoregulatory molecule(s) secreted by FAE may contribute to the differentiation and/or function of PP DCs, in the context of lymphepithelial interaction. To better understand such immunomodulators, we performed microarray analysis of the gene expression of FAE, followed by bioinformatics analysis using the recently introduced Reference Database for Immune Cells (RefDIC). Consequently, we found as a candidate for such immunomodulators pregnancy-specific glycoprotein 18 (Psg18), which is exclusively expressed in FAE.

Psg18 is a member of Psg family proteins. Psgs are the most abundant fetal proteins in the maternal bloodstream at the late stage of pregnancy (Lin et al., 1974). Their almost exclusive synthesis by trophoblasts of the haemochorial placenta in rodents and primates is well documented (Kromer et al., 1996; Lei et al., 1992). Multiple lines of evidence suggest that Psg modulates the maternal immune system to prevent rejection of the allotypic fetus. Low PSG levels are associated with certain pathological conditions in pregnancy (Silver et al., 1993). The administration of anti-PSG antibodies induced abortion in primates. The immunoregulatory function of PSG is underscored by the observation that PSG suppresses the proliferative response of peripheral blood lymphocytes (Harris et al., 1984). Furthermore, treatment of human monocytes or murine macrophages with recombinant PSG/Psg proteins induces the secretion of anti-inflammatory cytokines, including IL-10 and TGF- $\beta 1$ (Snyder et al., 2001). Likewise, recombinant Psg 18 and its N-terminal domain (Psg18N) selectively upregulate IL-10 but not IL$1 \beta$, TNF- $\alpha$ or IL- $12 p 40$ production in murine macrophages, even when costimulated with lipopolysaccharide, a potent inducer of proinflammatory cytokines (Wessells et al., 2000). To better understand the role of Psg 18 in the mucosal immune system, in the present study we characterized the expression and regulation of this molecule in FAE.

\section{Materials and Methods}

\section{Animal experiments}

Male BALB/c mice between 7 and 10 weeks old were obtained from Clea Japan Inc. (Tokyo, Japan) and maintained under specific- pathogen-free conditions at the animal facility of RIKEN RCAI. In certain experiments, $\mathrm{BALB} / \mathrm{c}$ mice were maintained under either germ-free (GF) or conventional (CV) condition at the animal facility of the University of Tokyo as described elsewhere (Narushima et al., 1999). Inoculation of GF-conditioned mice with a lethal dose $\left(1 \times 10^{3} \mathrm{cfu} /\right.$ mouse $)$ of enterohemorrhagic Escherichia coli O157:H7 or Bifidobacterium longum JCM1254 (1 $\times 10^{8} \mathrm{cfu} /$ mouse $)$ was performed as described previously (Itoh et al., 1988). FAE samples were collected at days 1 and 7 after $B$. longum inoculation, or at days 1 and 5 (instead of 7, since the mice die around day 7) after E. coli $\mathrm{O} 157$ inoculation. All animal experiments were carried out in accordance with the guidelines for the care and use of laboratory animals of RIKEN Yokohama Research Institute and of The University of Tokyo.

\section{Microarray and bioinformatics analysis}

Mouse genome-wide gene expression was examined using the Mouse Genome MOE430 2.0 Probe Array (Affymetrix, Santa Clara, CA). The procedure is basically the same as that described elsewhere (Hase et al., 2005). Data normalization was performed to scale the data so that the average intensity value on each array was 1 , by dividing each expression value by the median of the expression levels on each chip. The expression of Psg family members in various tissues and immune cells was analyzed with Reference Database for Immune Cells (RefDIC), a public bioinformatics database recently developed by RIKEN RCAI, Japan. The reliability of each microarray probe set for Psg was examined with a probe mapping tool in RefDIC, which predicts whether or not each probe set recognizes coding regions of target genes.

The transcription factor binding sites (TFBSs) of several Psg genes were predicted with GEMS launcher software (Genomatix, Munich, Germany). The DNA sequence 500 bp upstream and 100 bp downstream of the transcription start site of each Psg was subjected to the analysis.

\section{RT-PCR analysis}

Total RNA was extracted from FAE and small intestinal and colonic epithelium by an RNeasy Mini Kit (Qiagen, Valencia, CA), and the first-strand cDNA was synthesized with ReverTra Ace- $\alpha$ (TOYOBO, Osaka, Japan) using Oligo-dT 20 primer. All procedures were performed in accordance with the manufacturer's instructions. Real-time quantitative PCR was performed basically as described previously (Hase et al., 2003), using SYBR premix Ex Taq (Takara Bio, Otsu, Japan) and an iCycler ${ }^{\mathrm{TM}}$ PCR platform (Biorad). The amount of glyceraldehyde-3-phosphate dehydrogenase $(G A P D H)$ mRNA in each sample was quantified and used as internal control for comparison among the different samples. Primers used for amplification were as follows: 5'-AGCAACGAAGTCCATCATCAGAG-3' (forward) and 5'-AAGAGCCAACGGATGGAGATC-3' (reverse) for Psg18. GAPDH primers were described elsewhere. 


\section{In situ hybridization (ISH)}

DNA fragment corresponding to nucleotide positions 377-820 of mouse Psg18 cDNA (GenBank accession number: NM_011963) was amplified from FAE-derived cDNA by PCR using primers 5'-CTGTGGATCCAAAATGTCACACA-3' (forward) and 5'GATCTCGAGTCAGAGTTCGTAGG-3' (reverse). The PCR product was digested with $\mathrm{Bam} \mathrm{HI}$ and $\mathrm{XhoI}$ and was ligated into pcDNA3.0 digested with the same enzymes. Digoxigenin-labeled RNA probes were prepared by in vitro transcription with $\mathrm{T} 7$ or SP6 RNA polymerase and DIG RNA Labeling Mix (Roche Diagnostics, Penzberg, Germany). Paraffin-embedded PP sections were deparaffinized, treated with protease $\mathrm{K}$, and hybridized with 100 $\mathrm{ng} / \mathrm{ml}$ digoxigenin-labeled RNA probes at $60^{\circ} \mathrm{C}$ for $16 \mathrm{~h}$. Specific binding was detected by incubating with alkaline phosphataseconjugated anti-digoxigenin antibody (Roche Diagnostics) for $2 \mathrm{~h}$ at room temperature, and visualized with purple AP substrate overnight. The sections were counterstained with nuclear fast red.

\section{Immunohistochemistry}

Polyclonal anti-mouse Psg18 antibody were raised in two rabbits against a $\mathrm{COOH}$-terminal 16-amino acid peptide (WQAIAQHWYYYAVMVK) conjugated with BSA, and IgG fraction was purified by using protein A Sepharose (Pharmacia). The peptide sequence highly specific for Psg18 was selected, to minimize the cross-reactivity of the raised antisera to other Psg members. Paraffin-embedded sections of mouse PP, mesenteric lymph node, inguinal lymph node (as a representative of peripheral lymph node), spleen and lamina propria of small intestine were incubated with $0.5 \%$ blocking buffer (Roche) in PBS for $30 \mathrm{~min}$ at room temperature, followed by incubation with Psg18-specific antibody or control rabbit IgG in blocking buffer for 2 hours at room temperature. The binding of primary Ab was detected with Alexa488conjugated anti-rabbit IgG antibody (Invitrogen, Carlsbad, CA).

In a separate experiment, PP dissected from the small intestine was subjected to whole mount staining with biotin-conjugated hamster anti-mouse CD11c (clone HL3; BD) and the rabbit antimouse Psg18 antibody, followed by Cy5-conjugated streptavidin and Alexa488-conjugated anti-rabbit IgG antibody. The whole mount specimens were analyzed with a Delta Vision RT system (Applied Precision, Issaquah, WA).

\section{Preparation of PP DCs}

PPs were excised from the mouse intestinal wall and dissociated with collagenase solution containing $0.5 \mathrm{mg} / \mathrm{ml}$ collagenase (Nitta Gelatin), $0.5 \mathrm{mg} / \mathrm{ml}$ DNase I (Roche), 2\% FBS, $100 \mathrm{U} / \mathrm{ml}$ penicillin, $100 \mu \mathrm{g} / \mathrm{ml}$ streptomycin, and $20 \mathrm{mM}$ HEPES ( $\mathrm{pH}$ 7.2) in RPMI 1640 (Invitrogen Life Technologies) at $37^{\circ} \mathrm{C}$ for $15 \mathrm{~min}$ to obtain single cell suspension. The cell dissociation was repeated again in fresh collagenase solution. The single cell suspension was washed with RPMI 1640 twice and subjected to OptiPrep ${ }^{\circledR}$ density centrifugation ( $\rho=1.065 \mathrm{~g} / \mathrm{ml}$; Axis shield).

\section{Flow cytometric analysis}

Isolated mouse PP DCs were incubated in FACS buffer (2\% FCS in PBS) with anti-CD16/32 (clone 94; eBioscience) for 15min, and then to characterize cell populations, PP DCs were stained with FITC-conjugated mAbs against CD11c (HL-3); PE-conjugated mAbs CD3e (145-2C11), CD11b (M1/70) or B220 (RA3-6B2); and biotinylated mAbs against CD9 (KMC8) or Rat IgG2a in combination with streptavidin-PE-Cy7 (all from BD Pharmingen). The stained cells were analyzed using a FACSCalibur with CellQuest software (BD Bioscience).

\section{Results}

\section{Analysis of Psg transcript distribution in various tissues}

We have established a method to separate murine FAE and villous epithelium (VE) from PPs and small intestinal villi, respectively (Hase et al., 2005). Total RNAs prepared from FAE or VE were subjected to Affymetrix microarray analysis to search for immunomodulators that are specifically expressed in FAE. We found that the expression level of Psg18 was remarkably higher in FAE than in VE (Fig. 1). This observation is intriguing, considering that Psg family members are believed to be expressed exclusively in the placenta (Kromer et al., 1996).

Seventeen putative genes, Psg16-32, have been reported as murine Psg family members (McLellan et al., 2005). Among them, the probe sets for eight well-characterized genes (Psg 16-19, 21, 23, 28, and 32) are included in the Mouse Genome MOE430 2.0 gene chip. To examine the expression of Psg family members in various adult murine tissues including FAE, we performed bioinformatics analysis with RefDIC database search (http://refdic.rcai.riken.jp) (Fig. 1). This newly launched database contains 150 microarray analysis data of different tissues and immune cells prepared under various conditions, which enabled us to examine tissue- and cell-wide expression of target genes. Another feature of RefDIC is that the reliability of each probe can be inspected. Although the MOE430 2.0 gene chip contains approximately 45,000 probe sets, all of them do not necessarily correspond to the coding regions of their target genes. Indeed, some probe sets assigned to Psg matched the non-coding region of the target gene when compared to the reference sequence (Fig. S1). Furthermore, a probe set for Psg23 even showed the highest homology to an unrelated gene. Microarray analysis with these unfavorable probe sets may lead to bias of the data obtained. Therefore, only reliable probe sets whose sequences corresponded to the coding regions of target genes were selected and applied to the following analysis.

As shown in Fig. 1, predominant expression of Psg18 was observed in FAE but not in other cells or tissues. RTPCR analysis also confirmed that Psg18 is specifically 

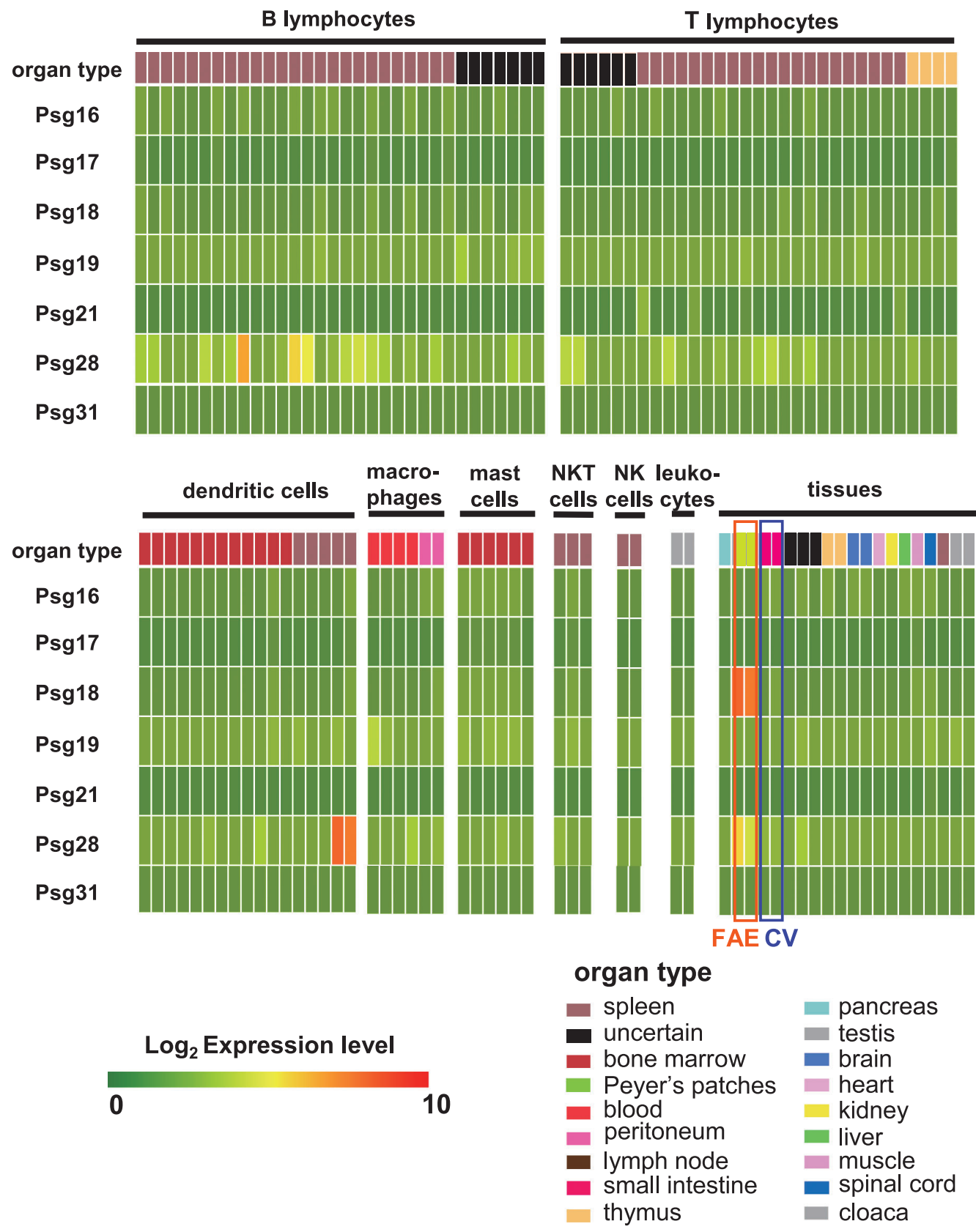

Fig. 1. Comprehensive analyses of the expression of Psg family members in various tissues and immune cells with RefDIC. Normalized data of the expression level of each gene are shown as a gradation from green (lowest) to red (highest). With regard to FAE and VE, two different RNA samples were obtained from GF- (left column) and CV- (right column) conditioned groups, respectively. Precise data and information on each sample are available on the web site (http://refdic.rcai.riken.jp/).

expressed in FAE as well as in the placenta tested as a positive control (Fig. 2A). Quantitative PCR analysis demonstrated that the expression level of Psg18 was more than 338- or 24-fold higher in FAE than in small intestinal or colonic epithelium, respectively (Fig. 2B). Psg28 was also detectable in FAE; however, its expression level was much lower than that of Psg18. On the other hand, Psg16, 17, 19,
21 and 32 were not expressed in the adult mouse tissues and cells examined here. Collectively, these data suggest that Psg 18 could be a novel FAE-specific marker in adult mice.

\section{Psg18-specific transcription-factor-binding domains}

The results so far suggest that TFBSs necessary for FAE- 
A)
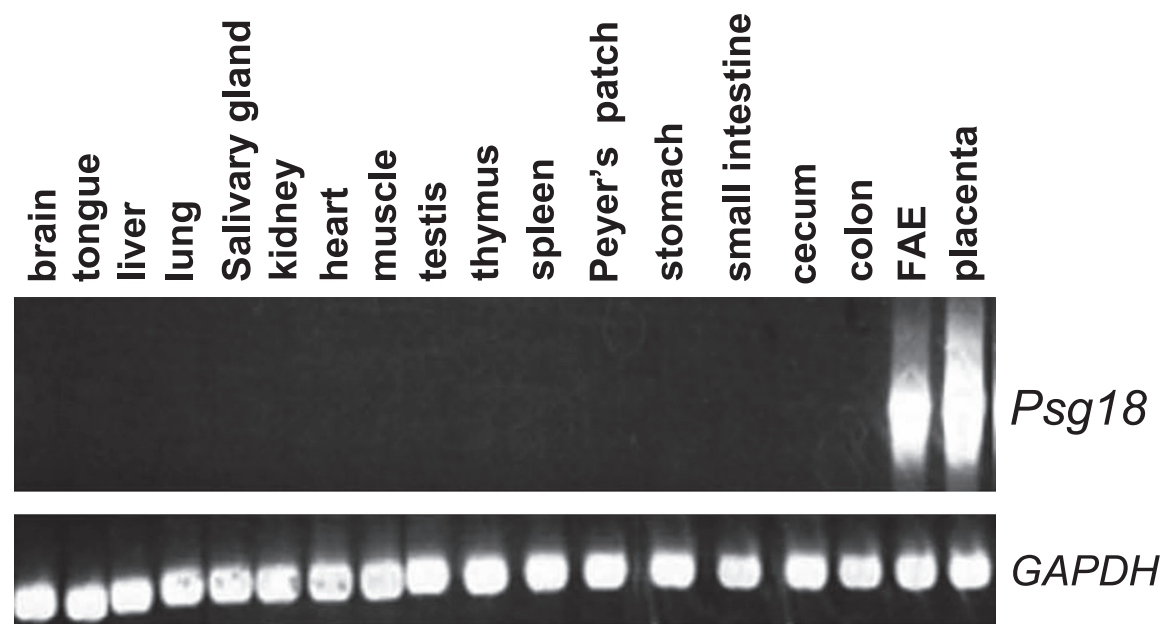

B)

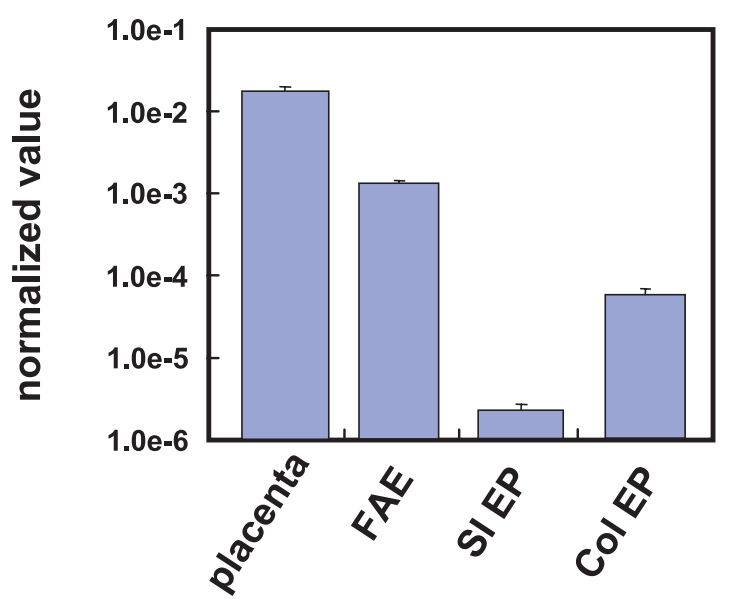

Fig. 2. PCR analysis of Psg 18 mRNA expression. A) The expression level of Psg 18 transcript in various tissues was determined by RT-PCR using specific primers for Psg18 and GAPDH as internal control. B) Psg18 mRNA expression in the placenta, FAE, small intestinal epithelium (SI Ep) and colonic epithelium (Col Ep) was analyzed by quantitative PCR. The mRNA expression levels of Psg18 were normalized to that of GAPDH. Data are mean $\pm \mathrm{SD}$ of three different samples.

specific expression may exist in 5'-UTR of Psg18. To predict such TFBSs, 5'-UTR of Psg18 was analyzed with GEMS launcher (Cartharius et al., 2005) and compared with other Psg family members. Nine TFBSs were commonly observed in all the Psg genes tested (Fig. 3A). On the other hand, four TFBSs, namely, the binding sites for Onecut homeodomain factor HNF6 (HNF6), Pancreas transcription factor 1 (PTF1), BTB/POZ transcription factor (BTBF), and Pregnane $\mathrm{X}$ receptor (PXRF), were found only in the Psg18 gene (Fig. 3B).

\section{Bacterial stimulation upregulates Psg18 expression in FAE}

It should be noted that the expression level of Psg 18 in FAE was almost twice as much in mice bred under $\mathrm{CV}$ condition than in mice bred under GF condition (normalized intensity values are 12.5 and 6.7, respectively). This suggests that Psg18 expression in FAE could be not only controlled autonomously but also affected by external factors such as bacterial colonization. To confirm this, mice bred under GF condition were inoculated with either B. longum or $E$. coli O157, which is a representative beneficial probiotic or enteropathogenic bacterium, respectively. Microarray data 


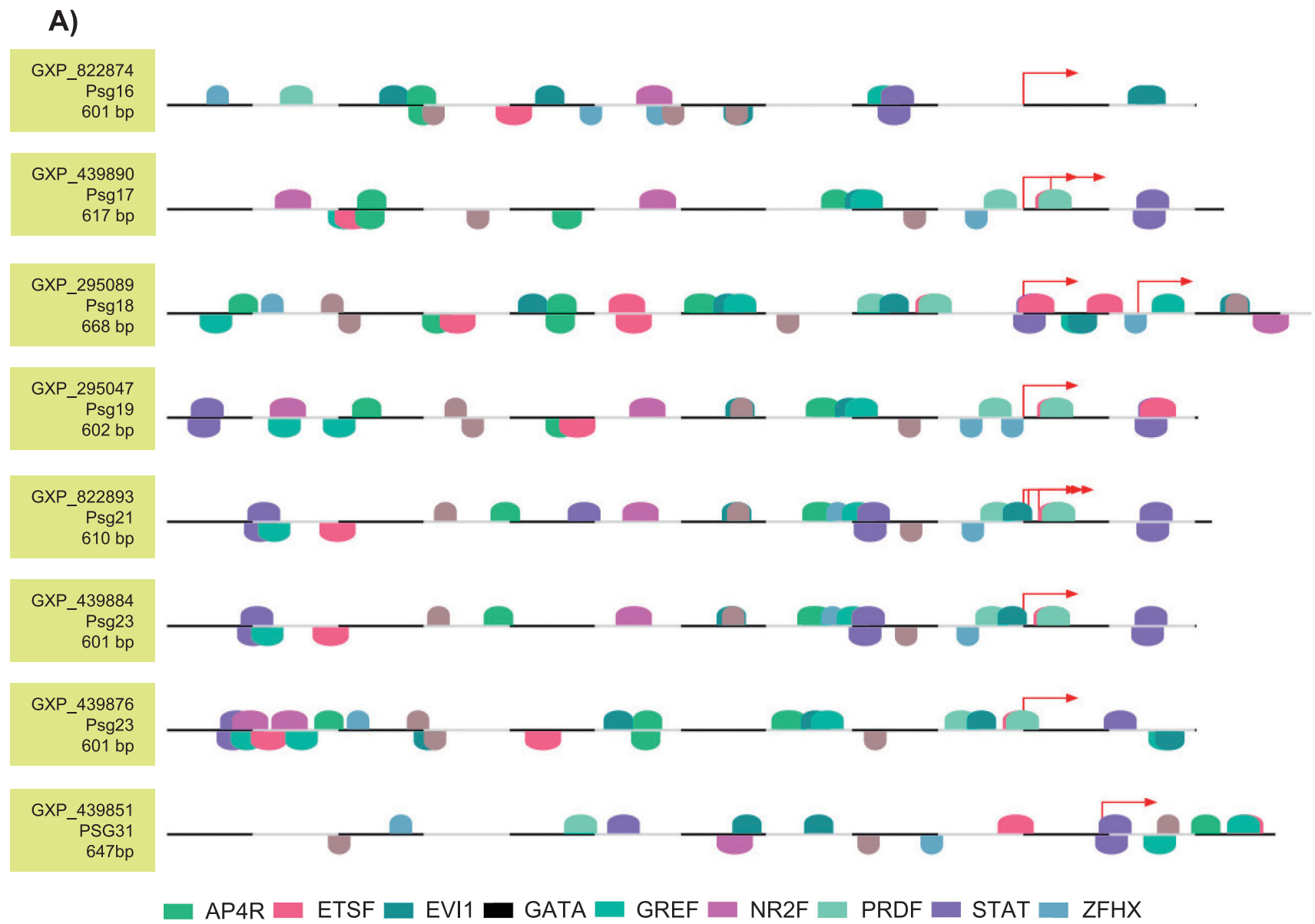

B)

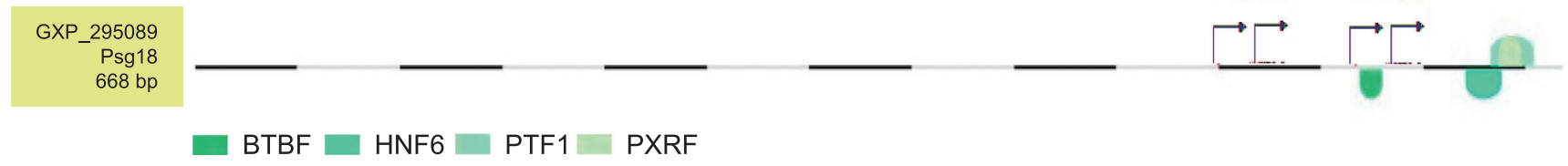

Fig. 3. Analysis of promoter regions of Psg genes with GEMS launcher. Red arrow indicates transcription start site. A) Nine TFBSs were commonly found in all the $P$ sg genes analyzed here. Different colors denote different transcription factor families: 1, AP4R; 2, ETSF; 3, EVI1; 4, GATA; 5, GREF; 6, NR2F; 7, PRDF; 8, STAT; 9, ZFHX. B) Four TFBSs were specifically found in the promoter region of Psg18 but not in other Psg family genes. 1, HNF6; 2, PTF1; 3, BTBF; 4, PXRF.

showed that Psg18 expression in FAE was 2- and 3-fold of that under GF condition at days 1 and 5 after E. coli $\mathrm{O} 157$ inoculation, respectively (Fig. 4). B. longum inoculation also upregulated $\operatorname{Psg} 18$ expression, although the effect was not as great as that of E. coli $\mathrm{O} 157$ inoculation. These data suggest that the expression of Psg 18 in FAE may be upregulated by exogenous stimuli such as bacteria.

\section{Psg18 is broadly expressed in FAE}

To confirm tissue and cell distributions of Psg18, we analyzed PP tissue sections for Psg18 expression by in situ hybridization. Positive signals were observed predominantly in FAE, but not in the intestinal villous epithelium or the lamina propria (Fig. 5, A-D). This result is consistent with microarray data and quantitative PCR analysis. Although FAE is heterogeneously composed of enterocytes, $\mathrm{M}$ cells, and goblet cells at a lesser frequency, Psg18 transcripts were distributed throughout FAE regardless of cell type. By contrast, no signal was observed on hybridization with a sense probe used as control. This observation further confirmed the FAE-specific expression of Psg18. 


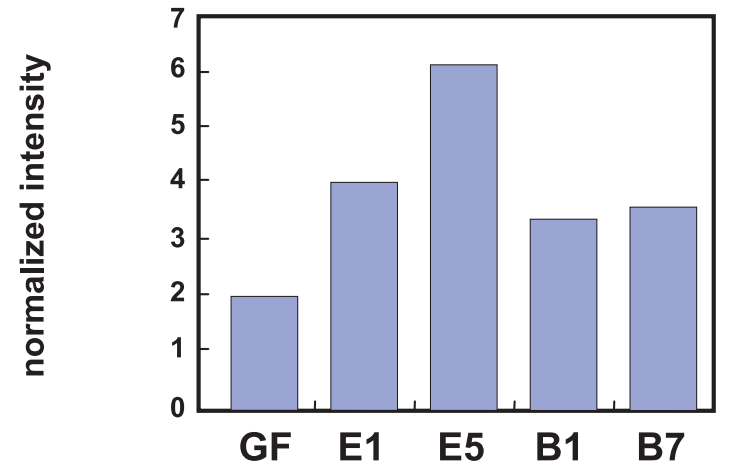

Fig. 4. Expression level of Psg18 in FAE of GF-conditioned mice before and after bacterial colonization. PP FAE samples were obtained from mice maintained under GF-condition (GF), 1 (E1) and 5 (E5) days after inoculation with $E$ coli $\mathrm{O} 157$, or 1 (B1) and 7 (B7) days after inoculation with $B$. longum. In each group, FAE samples from three mice were pooled and subjected to Affymetrix microarray analysis.
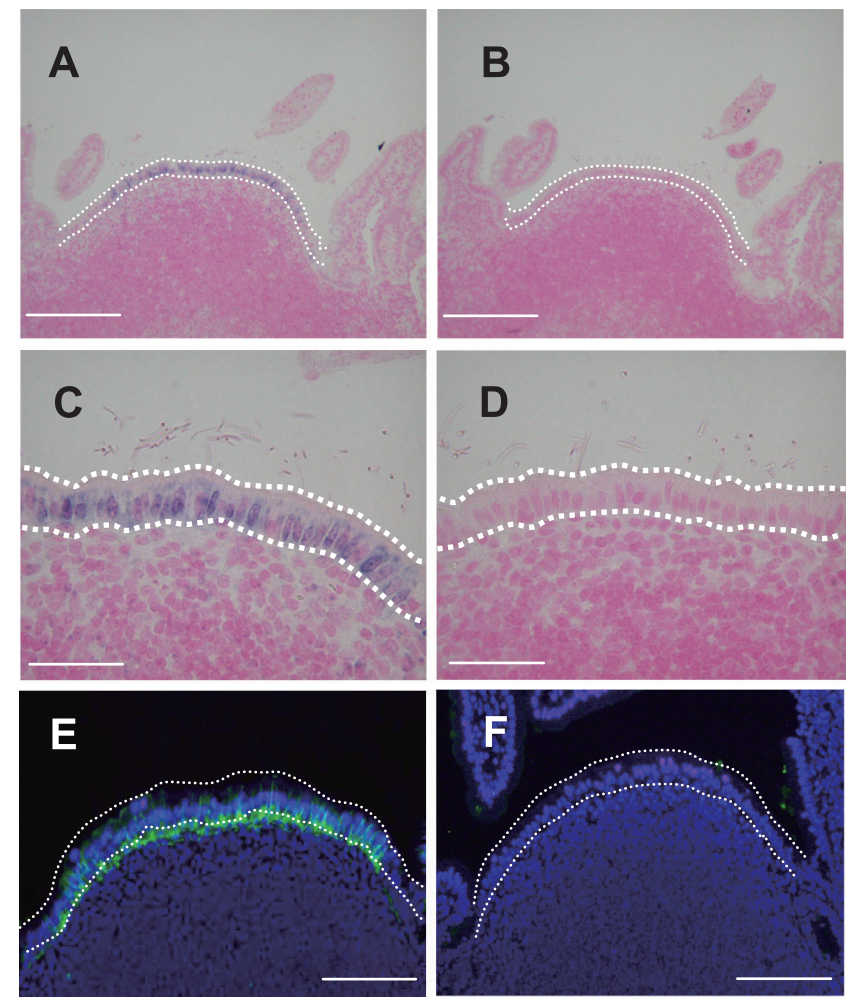

Fig. 5. Localization of Psg 18 mRNA and protein in mouse PPs. A-D: In situ hybridization was performed with Psgl8-specific antisense (A and C) or control sense (B and D) probe. E-F: Psg18 protein (green) was detected by immunostaining with Psg18-specific polyclonal antibody (E) or control rabbit IgG (F). DAPI (blue) was used for nuclear counterstaining. Scale bars indicate $100 \mu \mathrm{m}$.

\section{Psg18 protein is predominantly expressed in $F A E$}

To confirm the expression of Psg18 protein, we performed immunofluorescence staining of PPs with Psg18-specific polyclonal antibody. Consistent with ISH analysis data, Psg18 protein was detected in the FAE of PPs (Fig. 5E). Particularly strong expression of Psg18 was observed not only in the basolateral cytoplasm of FAE, but also in the extracellular space beneath FAE, which could reflect the deposition of secreted Psg 18 on the extracellular matrices in SED. Such staining closely resembles that of chemokines secreted from FAE toward SED in PPs (Iwasaki and Kelsall, 2001; Zhao et al., 2003). Additionally, Psg18 was expressed in FAE of colonic patches (Fig. S2A). In contrast, Psg18 was not detected in other lymphoid tissues including the thymus, mesenteric lymph node, peripheral lymph node and the lamina propria of the small intestine (Fig. S2C-F). These data further confirmed the FAE-specific expression of Psg18.

Immature DCs accumulate at the SED region in PPs (Johansson and Kelsall, 2005). To examine whether Psg18 deposited on the extracellular matrices beneath the FAE is recognized by DCs, we attempted double staining of Psg 18 and CD11c using whole mount specimens of PPs. The 3Dimaging with deconvolution microscopy technique demonstrated that the secreted Psg 18 protein reached the DC area of SED, and closely associated with the surface of DCs (Video 1).

We next analyzed the surface expression of CD9 on PP DCs. CD9 is the only receptor reported to bind the Psg family proteins including Psg17 and Psg19 (Waterhouse et al., 2002). Given that the highly conserved amino acid sequence and the domain structure among Psgs17, 18 and 19, we speculated that Psg18 might also bind to CD9 (Ha et al., 2005). We found that CD9 is highly expressed by CD $11 b^{+}$ DCs that is a major population of DCs residing in the SED in PP (Fig. 6) (Johansson and Kelsall, 2005). On the other hand, CD9 expression was very low and moderate, respectively, on $\mathrm{CD} 8 \alpha^{+}$and $\mathrm{B} 220^{+} \mathrm{DCs}$, the other DC subsets that have been reported to be present in the interfollicular region of PPs (Asselin-Paturel et al., 2003; Castellaneta et al., 2004; Iwasaki and Kelsall, 2001).

\section{Discussion}

In the present study, we found that Psg 18 mRNA and protein were expressed constitutively in FAE of PPs. The Psg family of glycoproteins is a subfamily of carcinoembryonic antigen (CEA), which also includes CEA-related cell adhesion molecules (CEACAMs). The domain structures of Psg are similar to those of CEACAMs; however, Psg lacks the transmembrane domain and is thereby secreted into the bloodstream. Membrane-anchored CEACAMs are widely expressed in both embryonic and adult tissues, and are asso- 


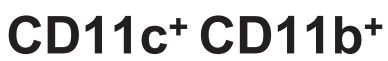

\section{CD11c ${ }^{+}$CD8 $\alpha^{+}$}

\section{CD11C $\mathrm{B}^{+} 220^{+}$}
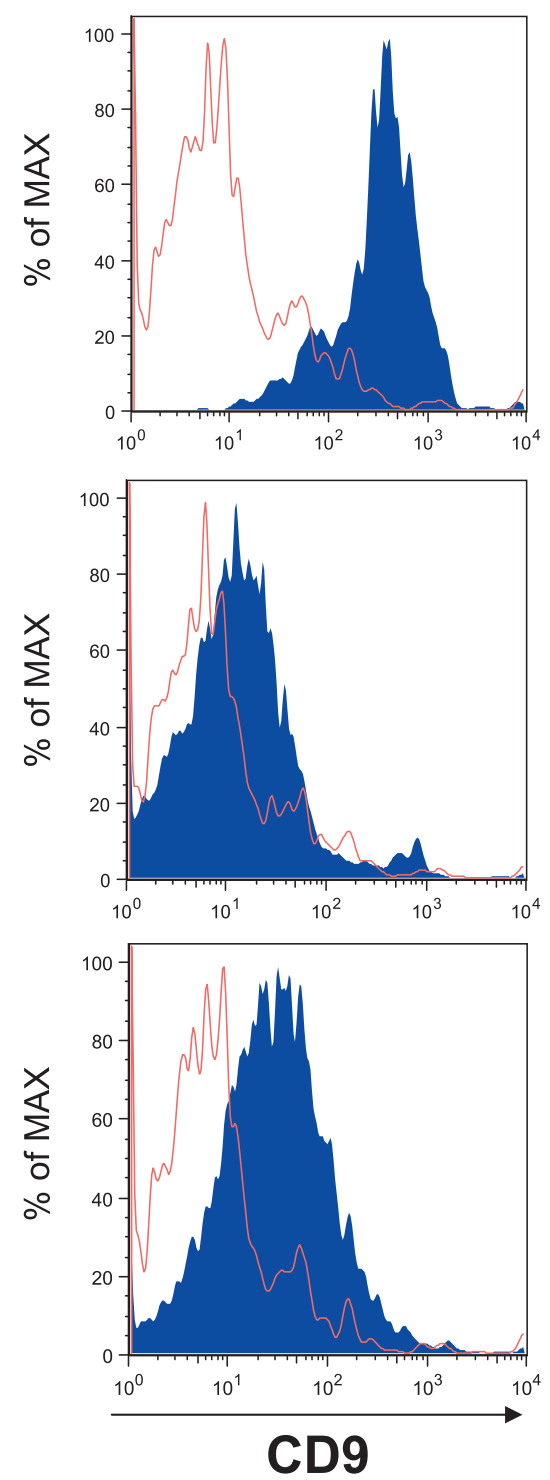

Fig. 6. FACS analysis of the CD9 expression level on DC subsets isolated from PPs. PP CD11 $\mathrm{c}^{+}$cells were stained with anti-CD11b, anti-CD8 $\alpha$ or antiB220 mAbs to distinguish three representative DC subpopulations. Surface expression of CD9 in each subpopulation was further analyzed. Filled histogram, CD9 staining; blank histogram, isotype control staining. Data were representative of three experiments.

ciated with cell growth, differentiation, and immune regulation (Gray-Owen and Blumberg, 2006; Han et al., 2001). On the other hand, Psg expression is almost exclusively restricted to the placenta, although a splicing variant of Psg16 that has only a partial N1 domain and no signal peptide is expressed in the brain (Chen et al., 1995a). Therefore, as far as we know, this is the first report of the production and secretion of full-length Psg in the adult mouse tissue.

It remains to be clarified how Psg18 expression is regulated in FAE. Analysis of the promoter regions of Psg with GEMS launcher has led to the identification of four possible TFBSs inherent to Psgl8 but not to the other Psg genes tested. These TFBSs may be responsible for the FAEspecific expression of Psg18. A previous study has shown that the expression of several molecules highly expressed in FAE is regulated by lymphotoxin- $\beta$ receptor (LT- $\beta$ R) signaling. For instance, the administration of LT- $\beta$ R-Fc fusion protein, which competitively blocks binding of $\mathrm{LT} \alpha / \beta$ to LT- $\beta$ R, reduces CCL20 expression in FAE (Rumbo et al., 2004). In addition, we recently found that clusterin, whose expression is known to be positively regulated by LT- $\beta$ R signaling (Huber et al., 2005), is constitutively expressed in FAE (Hase et al., 2005) (Hase et al., unpublished observation). We showed that bacterial colonization augmented Psg18 expression in FAE. This could be due to the direct 
stimulation of FAE through pathogen recognition receptors including toll-like receptors and NODs. Alternatively, it could also be possible that bacterial antigens stimulate antigen-presenting cells including DCs as well as B and T lymphocytes in SED and/or lymphoid follicles underneath FAE, which in turn, may enhance the expression of LT- $\beta$ R ligands on these cells (Zinetti et al., 1998). This could result in the upregulation of Psg18 in FAE, if Psg18 expression depends on the LT- $\beta$ R-mediated signaling pathway in analogy with CCL20 and clusterin (Huber et al., 2005; Rumbo et al., 2004).

The fetus is considered to be a kind of allograft in the maternal body; nevertheless, in most cases, immune rejection of the fetus does not occur (Trowsdale and Betz, 2006). A substantial body of evidence suggests that maternal immunity is skewed toward the anti-inflammatory Th2 condition during pregnancy, which protects the developing fetus from immune rejection. PSG released by the placenta plays a pivotal role in the induction of Th2 response (Snyder et al., 2001). This dogma could apply to the mucosal immune system as well, especially in the inductive sites of GALT represented by PPs. The bias toward Th2 response in PPs is essential for the production of secretory IgA and the tolerogenic response to commensal bacteria as well as food antigens (Mowat, 2003). It has been reported that the induction of Th2 response is mainly attributed to a particular subset of CD11 $\mathrm{b}^{+}$DCs residing in SED of PPs (Iwasaki and Kelsall, 2001; Sato et al., 2003). In this report, we found that Psg18 is secreted from FAE and is deposited in the SED region. In addition, $\mathrm{CD} 11 \mathrm{~b}^{+} \mathrm{DC}$ was found to highly express the Psg receptor, CD9. The binding of Psgs to CD9 has been reported to induce Th2 cytokines IL-10 and IL-6 as well as TGF- $\beta_{1}$ in human monocytes and murine macrophages $(\mathrm{Ha}$ et al., 2005). These observations raise the possibility that Psg18 may condition DCs to produce Th2 cytokines via CD9, although further investigation is required to test this hypothesis.

Acknowledgements. We wish to thank $\mathrm{H}$. Takatsu for his valuable suggestions. This study was supported in part by Grants-in-Aid for Young Scientists (B) (K.H.) and Scientific Research in Priority Areas (H.O. and K.H.) from the Ministry of Education, Culture, Sports, Science and Technology of Japan.

\section{References}

Asselin-Paturel, C., Brizard, G., Pin, J.J., Briere, F., and Trinchieri, G. 2003. Mouse strain differences in plasmacytoid dendritic cell frequency and function revealed by a novel monoclonal antibody. J. Immunol., 171: 6466-6477.

Cartharius, K., Frech, K., Grote, K., Klocke, B., Haltmeier, M., Klingenhoff, A., Frisch, M., Bayerlein, M., and Werner, T. 2005. MatInspector and beyond: promoter analysis based on transcription factor binding sites. Bioinformatics, 21: 2933-2942.

Castellaneta, A., Abe, M., Morelli, A.E., and Thomson, A.W. 2004. Identification and characterization of intestinal Peyer's patch interferonalpha producing (plasmacytoid) dendritic cells. Hum. Immunol., 65: 104-113.
Chen, D.S., Asanaka, M., Yokomori, K., Wang, F., Hwang, S.B., Li, H.P., and Lai, M.M. 1995a. A pregnancy-specific glycoprotein is expressed in the brain and serves as a receptor for mouse hepatitis virus. Proc. Natl. Acad. Sci. USA, 92: 12095-12099.

Chen, Y., Inobe, J., Marks, R., Gonnella, P., Kuchroo, V.K., and Weiner, H.L. 1995b. Peripheral deletion of antigen-reactive T cells in oral tolerance. Nature, 376: 177-180.

Gray-Owen, S.D. and Blumberg, R.S. 2006. CEACAM1: contactdependent control of immunity. Nat. Rev. Immunol., 6: 433-446.

Ha, C.T., Waterhouse, R., Wessells, J., Wu, J.A., and Dveksler, G.S. 2005. Binding of pregnancy-specific glycoprotein 17 to CD9 on macrophages induces secretion of IL-10, IL-6, PGE2, and TGF-beta1. J. Leukoc. Biol., 77: 948-957.

Han, E., Phan, D., Lo, P., Poy, M.N., Behringer, R., Najjar, S.M., and Lin, S.H. 2001. Differences in tissue-specific and embryonic expression of mouse Ceacam1 and Ceacam2 genes. Biochem. J., 355: 417-423.

Harris, S.J., Anthony, F.W., Jones, D.B., and Masson, G.M. 1984. Pregnancy-specific-beta 1-glycoprotein: effect on lymphocyte proliferation in vitro. J. Reprod. Immunol., 6: 267-270.

Hase, K., Murakami, M., Iimura, M., Cole, S.P., Horibe, Y., Ohtake, T., Obonyo, M., Gallo, R.L., Eckmann, L., and Kagnoff, M.F. 2003. Expression of LL-37 by human gastric epithelial cells as a potential host defense mechanism against Helicobacter pylori. Gastroenterology, 125: $1613-1625$.

Hase, K., Murakami, T., Takatsu, H., Shimaoka, T., Iimura, M., Hamura, K., Kawano, K., Ohshima, S., Chihara, R., Itoh, K., Yonehara, S., and Ohno, H. 2006. The membrane-bound chemokine CXCL16 expressed on follicle-associated epithelium and $\mathrm{M}$ cells mediates lympho-epithelial interaction in GALT. J. Immunol., 176: 43-51.

Hase, K., Ohshima, S., Kawano, K., Hashimoto, N., Matsumoto, K., Saito, H., and Ohno, H. 2005. Distinct gene expression profiles characterize cellular phenotypes of follicle-associated epithelium and m cells. DNA Res., 12: 127-137.

Huber, C., Thielen, C., Seeger, H., Schwarz, P., Montrasio, F., Wilson, M.R., Heinen, E., Fu, Y.X., Miele, G., and Aguzzi, A. 2005. Lymphotoxin-beta receptor-dependent genes in lymph node and follicular dendritic cell transcriptomes. J. Immunol., 174: 5526-5536.

Itoh, K., Matsui, T., Tsuji, K., Mitsuoka, T., and Ueda, K. 1988. Genetic control in the susceptibility of germfree inbred mice to infection by Escherichia coli O115a,c:K(B). Infect. Immun., 56: 930-935.

Iwasaki, A. and Kelsall, B.L. 1999. Freshly isolated Peyer's patch, but not spleen, dendritic cells produce interleukin 10 and induce the differentiation of T helper type 2 cells. J. Exp. Med., 190: 229-239.

Iwasaki, A. and Kelsall, B.L. 2001. Unique functions of CD11b+, CD8 alpha+, and double-negative Peyer's patch dendritic cells. J. Immunol., 166: 4884-4890.

Johansson, C. and Kelsall, B.L. 2005. Phenotype and function of intestinal dendritic cells. Semin. Immunol., 17: 284-294.

Kromer, B., Finkenzeller, D., Wessels, J., Dveksler, G., Thompson, J., and Zimmermann, W. 1996. Coordinate expression of splice variants of the murine pregnancy-specific glycoprotein (PSG) gene family during placental development. Eur. J. Biochem., 242: 280-287.

Lei, K.J., Sartwell, A.D., Pan, C.J., and Chou, J.Y. 1992. Cloning and expression of genes encoding human pregnancy-specific glycoproteins. J. Biol. Chem., 267: 16371-1638.

Lin, T.M., Halbert, S.P., and Spellacy, W.N. 1974. Measurement of pregnancy-associated plasma proteins during human gestation. J. Clin. Invest., 54: 576-582.

McLellan, A.S., Fischer, B., Dveksler, G., Hori, T., Wynne, F., Ball, M., Okumura, K., Moore, T., and Zimmermann, W. 2005. Structure and evolution of the mouse pregnancy-specific glycoprotein (Psg) gene locus. BMC Genomics, $\mathbf{6}: 4$.

Mowat, A.M. 2003. Anatomical basis of tolerance and immunity to intesti- 
nal antigens. Nat. Rev. Immunol., 3: 331-341.

Narushima, S., Itoh, K., Takamine, F., and Uchida, K. 1999. Absence of cecal secondary bile acids in gnotobiotic mice associated with two human intestinal bacteria with the ability to dehydroxylate bile acids in vitro. Microbiol. Immunol., 43: 893-897.

Rimoldi, M., Chieppa, M., Salucci, V., Avogadri, F., Sonzogni, A., Sampietro, G.M., Nespoli, A., Viale, G., Allavena, P., and Rescigno, M. 2005. Intestinal immune homeostasis is regulated by the crosstalk between epithelial cells and dendritic cells. Nat. Immunol., 6: 507-514.

Rumbo, M., Sierro, F., Debard, N., Kraehenbuhl, J.P., and Finke, D. 2004. Lymphotoxin beta receptor signaling induces the chemokine CCL20 in intestinal epithelium. Gastroenterology, 127: 213-223.

Sansonetti, P.J. 2004. War and peace at mucosal surfaces. Nat. Rev. Immunol., 4: 953-964.

Sato, A., Hashiguchi, M., Toda, E., Iwasaki, A., Hachimura, S., and Kaminogawa, S. 2003. CD11b+ Peyer's patch dendritic cells secrete IL6 and induce IgA secretion from naive B cells. J. Immunol., 171: 3684 3690.

Silver, R.M., Heyborne, K.D., and Leslie, K.K. 1993. Pregnancy specific beta 1 glycoprotein (SP-1) in maternal serum and amniotic fluid; preeclampsia, small for gestational age fetus and fetal distress. Placenta, 14: $583-589$.

Snyder, S.K., Wessner, D.H., Wessells, J.L., Waterhouse, R.M., Wahl, L.M., Zimmermann, W., and Dveksler, G.S. 2001. Pregnancy-specific glycoproteins function as immunomodulators by inducing secretion of IL-10, IL-6 and TGF-beta1 by human monocytes. Am. J. Reprod. Immunol., 45: 205-216.

Trowsdale, J. and Betz, A.G. 2006. Mother's little helpers: mechanisms of maternal-fetal tolerance. Nat. Immunol., 7: 241-246.

Waterhouse, R., Ha, C., and Dveksler, G.S. 2002. Murine CD9 is the receptor for pregnancy-specific glycoprotein 17. J. Exp. Med., 195: 277282.

Wessells, J., Wessner, D., Parsells, R., White, K., Finkenzeller, D., Zimmermann, W., and Dveksler, G. 2000. Pregnancy specific glycoprotein 18 induces IL-10 expression in murine macrophages. Eur. J. Immunol., 30: 1830-1840.

Zhao, X., Sato, A., Dela Cruz, C.S., Linehan, M., Luegering, A., Kucharzik, T., Shirakawa, A.K., Marquez, G., Farber, J.M., Williams, I., and Iwasaki, A. 2003. CCL9 is secreted by the follicle-associated epithelium and recruits dome region Peyer's patch $\mathrm{CD} 11 \mathrm{~b}+$ dendritic cells. $J$. Immunol., 171: 2797-2803.

Zinetti, M., Agyekum, S., Evans, T., Polak, J., and Cohen, J. 1998. The role of lipopolysaccharide, pro-inflammatory cytokines and bacterial superantigens in the transcriptional regulation of lymphotoxin alpha and beta in mouse splenocytes. Cytokine., 10: 940-947.

(Received for publication, May 2, 2007 and accepted, September 22, 2007) 
Psg18 Is Specifically Expressed in FAE

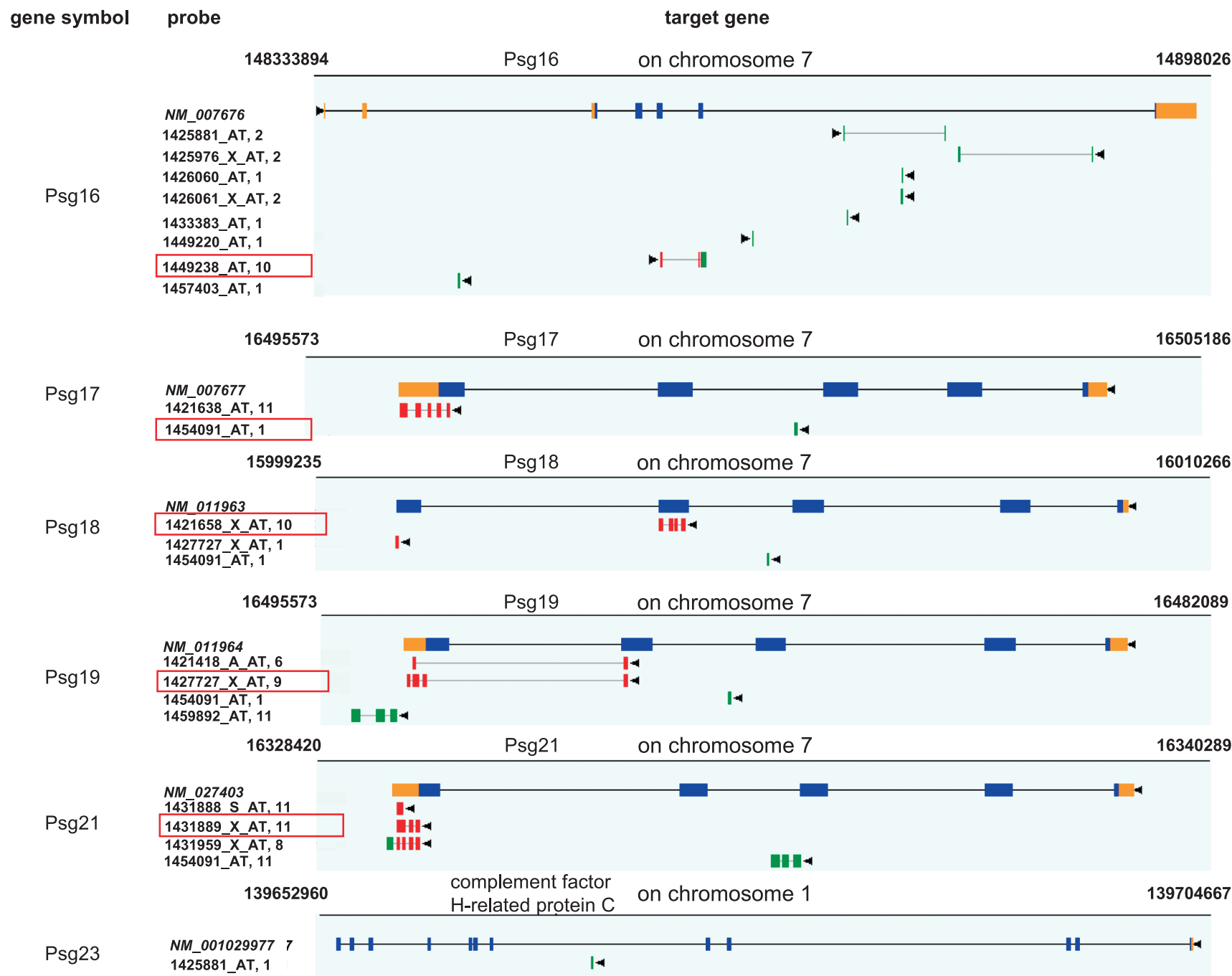

16075932

Psg28

NM_054063

$1421658 \times$ AT, 3 1425881_AT, 11

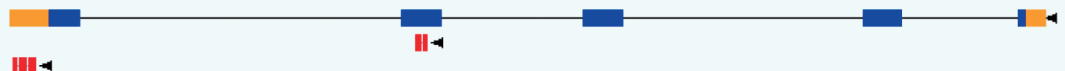

III-

on chromosome 7

15337454

Psg32 XR_000455

(Psg-ps1) XR_000338

$X R_{-} 000454$

1425712 AT, 1

1451616_AT, 10

15325710

Psg32

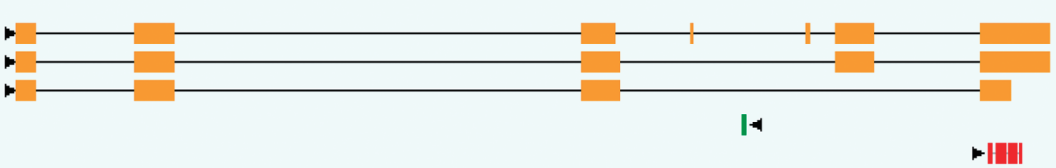

4

-CDS - UTR - not on Exon - Probe on Exon

Fig. S1. Psg probe mapping on chromosome. Affymetrix gene chip probes registered for Psg genes were surveyed with the probe mapping tool of RefDIC. The accession number of reference sequence for each gene was shown in italic. Coding sequences (CDS) and untranslated regions (UTR) on exons of each gene were indicated as blue and orange box, respectively. Of note is that the CDS of Psg32 has not been identified yet. Probe sequences that correspond to those of the exon or intron of target gene were indicated with red or green bars, respectively. The probe sets highlighted with red boxes were used for the following experiments. 


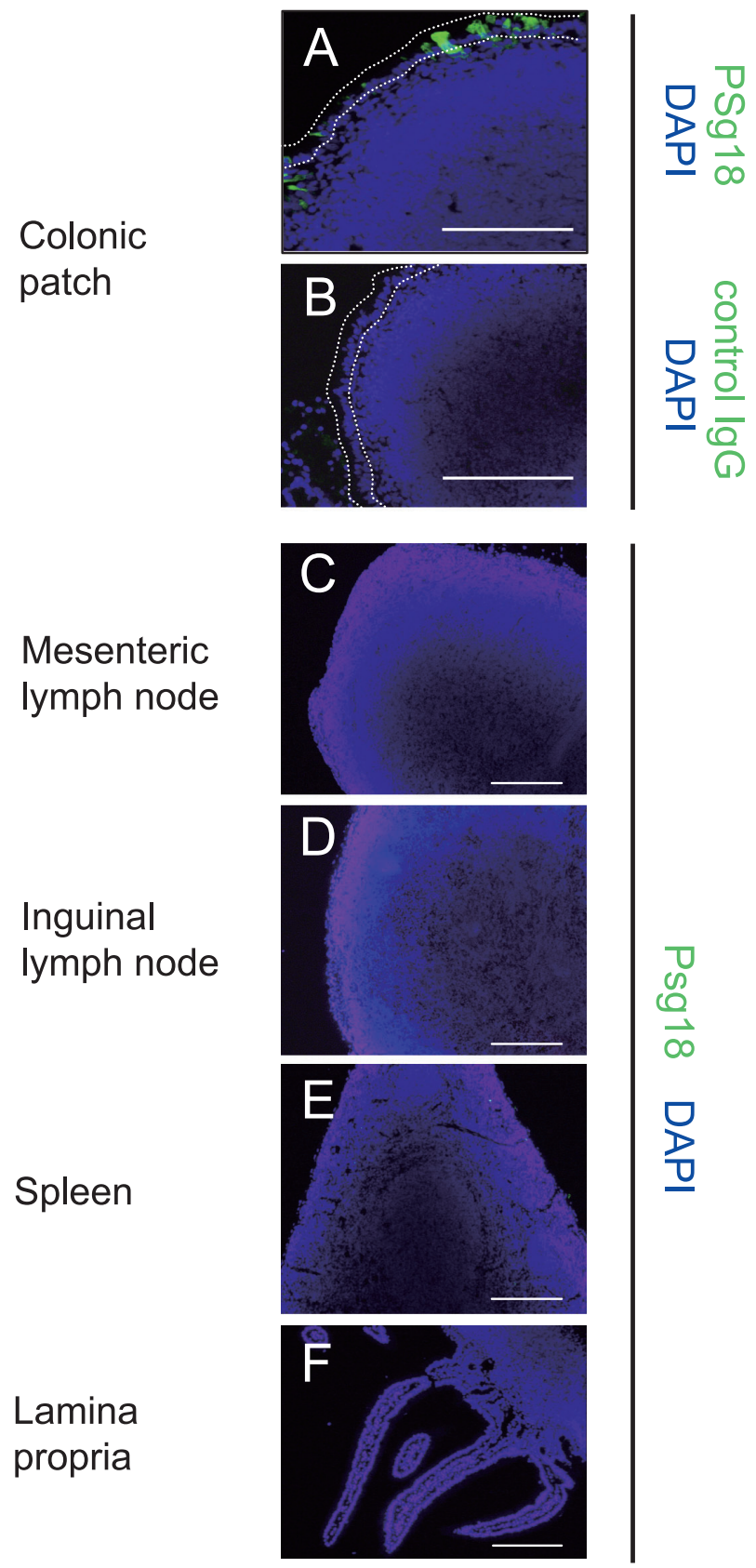

Fig. S2. Immunofluorescence staining of immune tissues for Psg18 expression. Psg18 protein (green) was stained with Psg18-specific polyclonal antibody (A, C-F) or control rabbit $\operatorname{IgG}(\mathrm{B})$ as described in the Materials and Methods. DAPI (blue) was used for nuclear counter staining. Scale bars indicate $100 \mu \mathrm{m}$. 